\title{
ARTICLE \\ Effects of substance misuse on reward-processing in patients with attention-deficit/hyperactivity disorder
}

\author{
Maria Paraskevopoulou (iD ${ }^{1,2}$, Daan van Rooij $\mathbb{D}^{3}{ }^{3}$, Albert Batalla ${ }^{4}$, Roselyne Chauvin ${ }^{3}$, Maartje Luijten ${ }^{5}$, Aart H. Schene $^{1,2}$, \\ Jan K. Buitelaar $\mathbb{i D}^{2,6}$ and Arnt F. A. Schellekens ${ }^{1,7}$
}

\begin{abstract}
Attention-Deficit/Hyperactivity Disorder (ADHD) and Substance Use Disorder (SUD) often co-occur and are associated with treatment resistance. Both disorders are characterized by similar reward-processing deficits with decreased striatal responses to reward anticipation, though literature is inconsistent. It is unclear whether substance misuse exaggerates reward-processing deficits observed in ADHD. The aim of this study was to examine substance misuse effects on reward-processing in ADHD. Functional MRI data in a Monetary Incentive Delay (MID) task from a multi-site study were compared across ADHD groups with and without substance misuse (ADHD + SM and ADHD-only, respectively) and healthy controls ( $n=40 /$ group, 74 males and 46 females, aged 13.7-25.9 years). Substance misuse was defined as misuse of alcohol, nicotine, or drugs. Groups were matched with presence/ absence of parental SUD to avoid interference with SUD trait effects. Compared to ADHD-only and controls, ADHD + SM showed hyperactivation in putamen during reward anticipation. Compared to controls, the ADHD groups showed hypoactivation in motor/ sensory cortices and hyperactivation in frontal pole and OFC during reward outcome. ADHD + SM also showed hyperactivation in frontal pole during neutral outcome. Moreover, ADHD + SM patients showed higher callous-unemotional (CU) traits that were positively correlated with putamen responses to reward anticipation. Our results show distinct condition-independent neural activation profile for ADHD + SM compared to ADHD-only and controls. Effects of comorbid substance misuse and variability of its prevalence across ADHD studies might have contributed to inconsistencies in ADHD literature. Contrasted with findings for rewardprocessing in SUD literature, results potentially suggest distinct underlying mechanisms for SUD subgroups with different characteristics, like antisocial/psychopathic traits.
\end{abstract}

Neuropsychopharmacology (2021) 46:622-631; https://doi.org/10.1038/s41386-020-00896-1

\section{INTRODUCTION}

Attention-Deficit/Hyperactivity Disorder (ADHD) is a common neurodevelopmental disorder that persists into adulthood for a considerable proportion of patients [1,2]. Adolescents and adults with $A D H D$ are at increased risk for developing Substance Use Disorder (SUD) [3-5], with prevalence rates up to $58 \%[6,7]$, contrasted with prevalence rates of $4-5 \%$ in the general population [8]. ADHD patients with comorbid SUD start substance use at an earlier age, progress more rapidly from recreational use to SUD [9] and respond poorly to SUD and ADHD treatment, compared to SUD-only [4, 9] and ADHD-only patients [10], respectively. This suggests that patients with ADHD and comorbid SUD represent a particularly severe and treatment resistant subgroup. Hence, investigating mechanisms contributing to this common comorbidity and treatment resistance is of paramount importance.

Patients with ADHD and SUD share several phenotypic characteristics. For instance, impulsivity is one of the core symptoms of ADHD [11, 12] and is also commonly observed in SUD patients $[13,14]$. At cognitive level, impulsivity consists of multiple components that are linked to both patient groups [15]. One of these components is (elevated) reward-seeking [15] that refers to abnormalities in reward-processing and is associated with altered reward sensitivity [16]. The cortico-basal ganglia circuit, and particularly the ventral striatum (VS), is thought to mediate reward-processing [17]. During adolescence, this pathway undergoes substantial developmental changes [18], potentially predisposing adolescents to reward-seeking behaviors. Several studies have investigated the role of this pathway in reward-processing deficits observed in ADHD and SUD patients.

Interestingly, ADHD literature shows different developmental trajectories of striatal maturation in patients compared to controls $[19,20]$. Moreover, meta-analysis of $\mathrm{fMRI}$ studies on neural activation during reward anticipation in a Monetary Incentive Delay (MID) task in ADHD patients showed VS hypoactivation for patients compared to controls [21]. This finding was replicated by a subsequent study that additionally found a positive association between VS anticipatory activation and parent-reported reward sensitivity for offspring [22]. In contrast, two studies showed nonsignificant VS hyperactivation for ADHD patients compared to

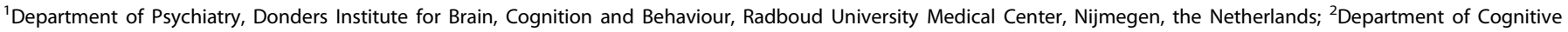

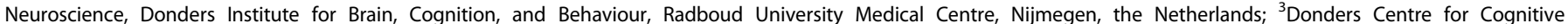

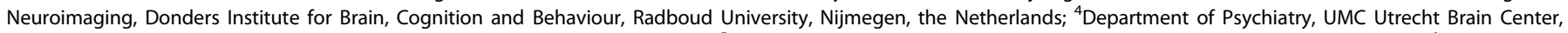

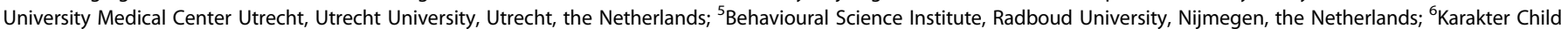
and Adolescent Psychiatry, Nijmegen, the Netherlands and ${ }^{7}$ Nijmegen Institute for Scientist Practitioners in Addiction, Nijmegen, the Netherlands Correspondence: Maria Paraskevopoulou (maria.paraskevopoulou@radboudumc.nl)
}

Received: 9 January 2020 Revised: 13 September 2020 Accepted: 12 October 2020 Published online: 12 November 2020 
controls during reward anticipation [23, 24]. Some studies also examined group differences during reward outcome showing either VS hyperactivation [24, 25] or no group differences [26]. Meta-analysis on reward-processing deficits in SUD patients showed that, compared to controls, SUD patients exhibited VS hypoactivation during reward anticipation, followed by VS hyperactivation during reward outcome in a MID task [27]. Similar findings have been observed in individuals with recreational drug and tobacco use $[28,29]$. Moreover, VS hyperactivation, predicted early-onset substance use in adolescents at high risk for SUD [30].

Where in ADHD impulsivity is thought to represent one of the core symptoms of the disorder, in SUD it is seen as both risk factor for and consequence of substance use. For instance, animal studies show that chronic drug administration results in alterations within the dopamine system, associated with various impulsivity components [31-37]. On the other hand, Belin et al. showed that high impulsivity predicted subsequent compulsive drug-taking in rats [38]. High impulsivity as part of ADHD is thus thought to mediate the increased risk for SUD in ADHD patients [39]. Moreover, most of the genetic liability shared between ADHD and alcohol use disorder (AUD) was found to be also shared with Conduct Disorder (CD) [40]. The callous-unemotional (CU) component of psychopathy (i.e., CU traits) [41] is suggested to be a modifier to CD diagnosis [42]. Impulsivity in ADHD-SUD comorbidity might thus reflect both effects of chronic substance use and shared trait effects. However, it's not clear whether CU traits are also associated with the impulsive behaviors observed in ADHD-SUD comorbidity.

To the best of our knowledge, there are no studies in ADHD patients examining effects of substance misuse on rewardprocessing. This study aims to address this question comparing neural responses to reward and neutral anticipation and outcome during a MID task between ADHD patients with and without substance misuse. Based on the results of a large number of ADHD studies, we hypothesized VS hypoactivation during reward anticipation, followed by VS hyperactivation during reward outcome for ADHD patients without substance misuse compared to controls. Furthermore, given the same direction of ADHD and SUD effects, we expected additive effects of substance misuse on reward-processing deficits in ADHD patients. More specifically, we hypothesized more pronounced VS hypoactivation during reward anticipation and VS hyperactivation during reward outcome for ADHD patients with comorbid substance misuse, compared to those without and to controls. Finally, we explored the association between $\mathrm{CU}$ traits and neural activation during the task.

\section{MATERIALS AND METHODS}

Participants

Participants of this study were part of the NeurolMAGE cohort [43], a longitudinal study that recruited ADHD and control families and focused on cognitive, neural, and genetic correlates of ADHD. Additional data on substance use was collected. NeurolMAGE was approved by the regional ethics committee (Centrale Commissie Mensgebonden Onderzoek: CMO Regio Arnhem Nijmegen; 2008/ 163; ABR: NL23894.091.08) and the medical ethics committee of the VU University Medical Center. Detailed description of the project, including obtaining the required informed consents, recruitment, and ADHD diagnostic procedure, can be found in Supplement 1 and the main design paper of NeuroIMAGE [43].

The present study included ADHD offspring from ADHD families and (unaffected) offspring from control families. Unaffected offspring from ADHD families were excluded $(n=92)$. For more detailed description of exclusion criteria see Supplement 1. After matching the groups with relevant covariates, the final sample of our study consisted of an ADHD group without substance misuse (ADHD-only, $n=40$ ), an ADHD group with substance misuse (ADHD + SM, $n=40)$, and a control group $(n=40)$. In total, our dataset included offspring from 105 families: 91 families with 1 offspring, 13 families with 2 offspring and 1 family with 3 offspring. Our data were also part of that included in Von Rhein et al. [24]. Sample characteristics are summarized in Table 1. Age and IQ distributions are included in Figs. S10 and S11.

\section{Instruments/measurements}

$A D H D$. The diagnostic algorithm for ADHD included assessment with the Schedule for Affective Disorders and Schizophrenia for School-Age Children-Present and Lifetime Version (K-SADS) [44] and Conners' ADHD questionnaires completed by parents, teachers, and participants $[45,46]$. The diagnostic algorithm is described in detail in Supplement 1 and in the main design paper of NeurolMAGE [43].

Substance use. Substance use was examined with the Dutch version of the Self-Reported Delinquency Scale (SRD) [47, 48]. Daily alcohol or nicotine use or (at least) weekly drug use indexed substance misuse [49]. Participants with missing data in at least one of the scales of the questionnaire (i.e., alcohol, drug, or tobacco) were excluded from the study $(n=15)$.

Parental substance use was assessed with the Alcohol Use Disorders Identification Test (AUDIT) [50], the Drug Abuse Screening Test (DAST) [51], the Fagerstrom Test for Nicotine Dependence (FTND) [52] and the Timeline Follow Back (TLFB; [53]; Supplement 1). Positive family history of SUD $(\mathrm{FH}+)$ was identified provided alcohol, drug, or nicotine dependence was present in at least one of the parents. Negative family history of SUD (FH-) was indicated for absence of alcohol, drug, and nicotine dependence in both parents. Participants were excluded from the study in case data on substance use were absent for at least one parent unless the data of the other parent indicated SUD and thus $\mathrm{FH}+$.

To avoid interference with familial trait effects, all groups were matched perfectly with family history of SUD (FH). To prevent the influence of additional confounders, all groups were matched optimally with age, and the ADHD groups were matched perfectly with sex (i.e., biological sex at birth). Matching was conducted with Matchlt in $R$ ( $R$ version 3.6.2; Rstudio version 1.2.5033) [54, 55].

Callous-unemotional (CU) traits. CU traits were assessed with the self-report Inventory of Callous-unemotional traits (ICU) [56]. This consisted of three subscales: uncaring, callousness, and unemotional. ICU has been validated in samples of adolescents and young adults, with construct consistency $a=0.74-0.85$ for total ICU, $a=0.70-0.84$ for uncaring, $a=0.71-0.88$ for callousness, $a=$ $0.45-0.60$ for unemotional $[56,57]$. Moreover, self-report ICU has previously been used for ADHD [58].

Monetary Incentive Delay (MID) task

Reward-processing was measured with an adapted version of the MID task $[24,59,60]$ (Fig. S1), consisting of one 12-min block with 25 reward, 25 neutral, and 25 trials without events. Reward and neutral trials contained presentation of a cue (jittered interval 3.5-8.5 s) that indicated trial type (i.e., reward or neutral), followed by the presentation of the target $(270-500 \mathrm{~ms})$. Time interval between trials was fixed at $5000 \mathrm{~ms}$. Participants were asked to respond to the target as soon as possible by pressing a button. This was followed by a feedback screen indicating the outcome of the trial. Outcome in reward trials included the gain of a monetary reward (i.e., 20 cents) for button presses within the response window, while neutral trials included no monetary reward gain. The response window for a correct trial was adapted in the next trial based on participants' performance, with an expected hit rate of $33 \%$. A detailed description of the task can be found in the paper of Von Rhein et al. [24].

Behavioral variables were mean reaction time (RT) and intraindividual coefficient of variation (ICV, defined as SD of RT/mean RT) in reward and neutral conditions. Trials with no or premature 


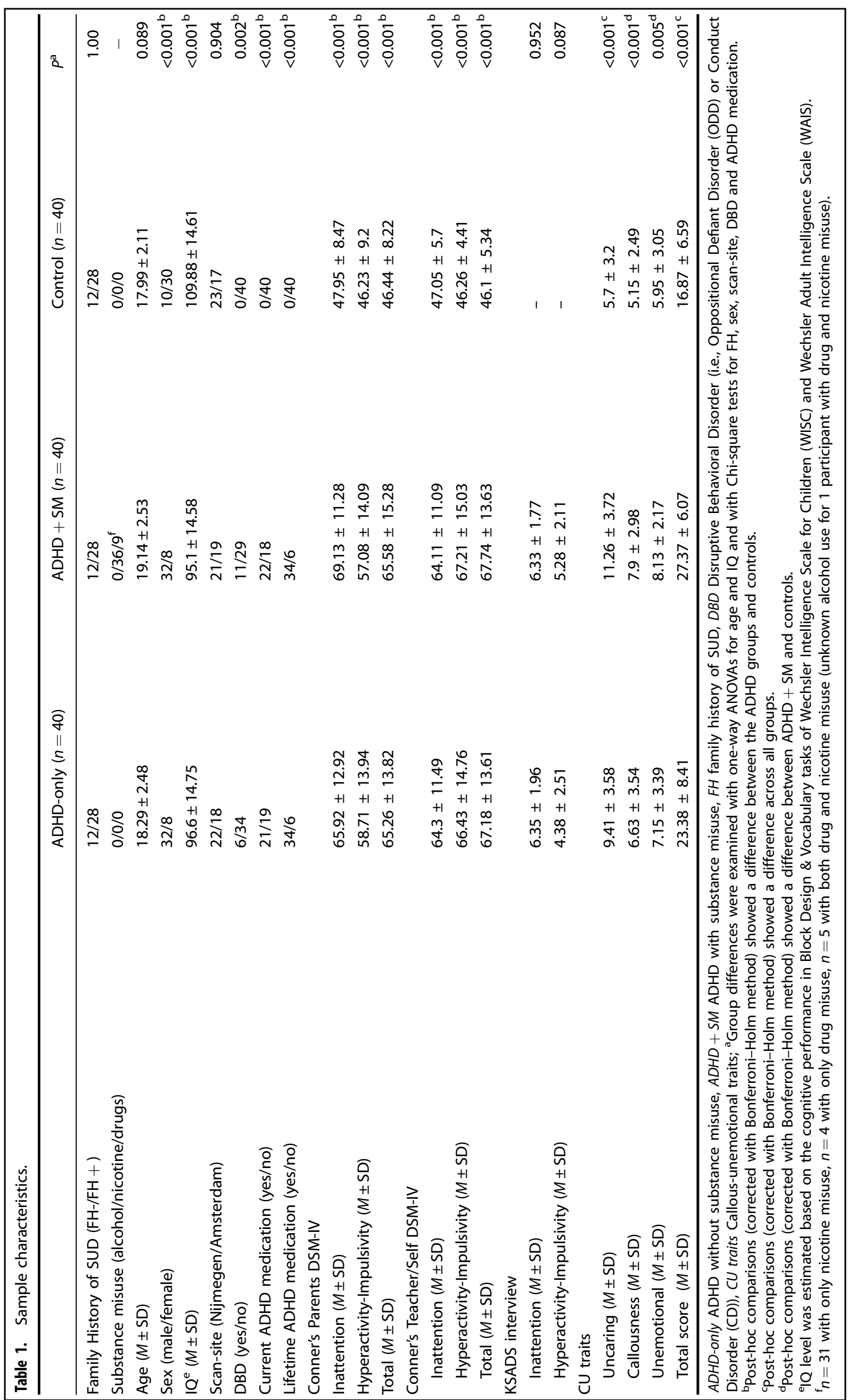


Table 2. Descriptive statistics of the behavioral data.

\begin{tabular}{|c|c|c|c|c|c|}
\hline Dependent Variables & ADHD-only & $\mathrm{ADHD}+\mathrm{SM}$ & Control & $p$ value & Significant contrasts ( $p$ value) \\
\hline Reward condition & $299.14 \pm 34.66$ & $281.36 \pm 36.21$ & $284.82 \pm 27.59$ & 0.02 & a $(0.018)$ \\
\hline \multicolumn{6}{|c|}{ Intra-individual Coefficient of Variation $(\mathrm{M} \pm \mathrm{SD})$} \\
\hline Reward condition & $0.17 \pm 0.06$ & $0.19 \pm 0.05$ & $0.17 \pm 0.06$ & 0.155 & ns \\
\hline
\end{tabular}

responses (i.e., RT $<100 \mathrm{~ms}$ or button press prior to target onset) or trials with more than one button presses were excluded from the calculation of the mean RT.

\section{Analyses}

Behavioral data analysis. The log10-transformation to normality was applied for variables that were not normally distributed. Task effect and group differences in RT and ICV were examined using separate two-way mixed ANCOVAs in R (R version 3.6.2; Rstudio version 1.2.5033) [54], with condition as the within-subjects independent variable (two levels: reward and neutral), group as the between-subjects independent variable (three levels: ADHDonly, ADHD + SM, controls) and FH, age, sex and scan-site as covariates. Significant group differences (i.e., $p<0.05$ ) were followed by post-hoc comparisons for pairwise differences and Bonferroni-Holm correction for multiple comparisons.

fMRI data analysis. MRI data acquisition and preprocessing are described in Supplement 1. For first-level analysis, statistical parametric maps were estimated with a General Linear Model (GLM) in FSL-FEAT $[61,62]$. The model included six regressors of interest (i.e., onset times for cues and onset times for hits and misses in reward and neutral trials) and five regressors of no interest (i.e., onset times for targets in reward and neutral trials and for cue, target, and outcome for trials with no, premature or multiple responses). Temporal derivatives of the regressors were also added to the model. All regressors were convolved with a canonical hemodynamic response function (HRF). Contrasts of interest consisted of reward anticipation (i.e., onset time for cues in reward trials), neutral anticipation (i.e., onset time for cues in neutral trials), reward outcome (i.e., onset times for reward hitsonset times for reward misses), neutral outcome (i.e., onset times for neutral hits-onset times for neutral misses), reward minus neutral anticipation and reward minus neutral outcome. The included contrasts intended to go beyond traditional reward minus neutral contrasts examining separately the reward and neutral conditions, in order to define where the actual differences across groups lie. First-level activation maps were spatially normalized into each participant's native space and subsequently transformed into MINI152 standard space for group-level analysis.

Whole-brain analysis. For each contrast, group-level analysis was performed with a Mixed-Effects Model with FSL flame [61, 62]. Ttests for group activation maps and an F-test contrast for differences across the three groups were included in the model. Mean-centered FH, age, sex, and scan-site were also added as regressors of no interest. Z-statistical images were thresholded with a cluster forming threshold of $Z>2.6$ and a family-wise corrected cluster significance threshold of $p<0.05$. Mean activation parameters ( $\beta$-values) of significant clusters were extracted for each participant, using the fslmeants function [61, 62], and posthoc comparisons with Bonferroni-Holm correction were performed in $R$ ( $R$ version 3.6.2; Rstudio version 1.2.5033) [54].
Region of interest (ROI) analysis. Based on a priori interest in VS activity, we created a nucleus accumbens (NAcc) region of interest (ROI) mask in FSL using the Harvard-Oxford subcortical structural atlas $[61,62]$. Using the fslmeants function [61, 62], individual $\beta$ values were extracted from the masked first-level statistical parametric maps for reward minus neutral anticipation and outcome and were used in separate one-way ANCOVAs to examine group differences in VS activity in these contrasts. $\mathrm{FH}$, age, sex, and scan-site were included as covariates.

Sensitivity analysis. To make sure that group differences of the main analysis were not driven by other confounding factors we did not account for, we conducted sensitivity analyses with separate linear mixed-effects models in $\mathrm{R}$ ( $\mathrm{R}$ version 3.6.2; Rstudio version 1.2.5033) [54] for each behavioral variable and $\beta$-values from each significant cluster. Factors added consisted of familiality (randomeffects factor; random intercept model), IQ, current ADHD medication usage, and presence of comorbid Disruptive Behavioral Disorder (DBD; Oppositional Defiant Disorder (ODD) or CD) [15].

Post-hoc exploratory Analysis. We performed multiple linear regression (with $A D H D+S M$ as reference group) in $R$ ( $R$ version 3.6.2; Rstudio version 1.2.5033) [54] to examine the relationship of the most important findings in the whole-brain analysis with ADHD severity and CU traits, which have been previously associated with elevated SUD prevalence [63]. Cluster activation was used as outcome variable and CU traits-group interaction and ADHD severity-group interaction as predictor variables in the same model. ADHD severity was measured with scores in hyperactivity-impulsivity and inattention subscales of the Conner's parents and teachers/self rating scales (i.e., average CPRS and CTRS scores) $[45,46]$. CU traits were assessed with scores in the uncaring, callousness, and unemotional subscales of ICU [56]. Regression coefficients were significant for $p<0.005$ (corrected for multiple comparisons).

\section{RESULTS}

Behavioral results

Descriptive statistics are summarized in Table 2 and are visually presented in Fig. S2. There was a main effect of condition on RT $(p<0.001)$, with shorter RTs for reward compared to neutral trials. We also found a main effect of group on RT $(p=0.02)$, with shorter RTs for ADHD + SM compared to ADHD-only patients $(p=0.018)$. No group-condition interaction was found $(p=0.283)$. Moreover, there was a main effect of condition on ICV $(p<0.001)$, with shorter ICV for reward compared to neutral trials, but no group differences ( $p=0.137)$ or group-condition interaction $(p=0.242)$. Covariate effects are described in Supplement 2.

fMRI results

Whole-brain analysis. Group activation maps during reward, neutral and reward minus neutral anticipation and outcome 


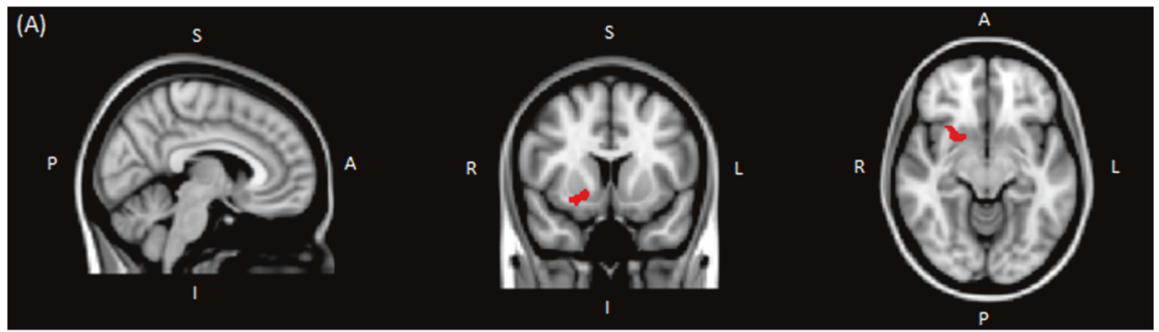

(B)

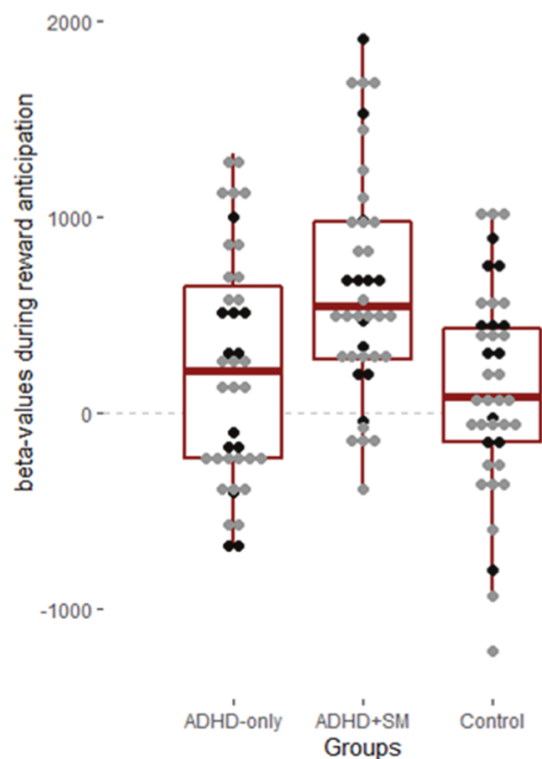

(C)
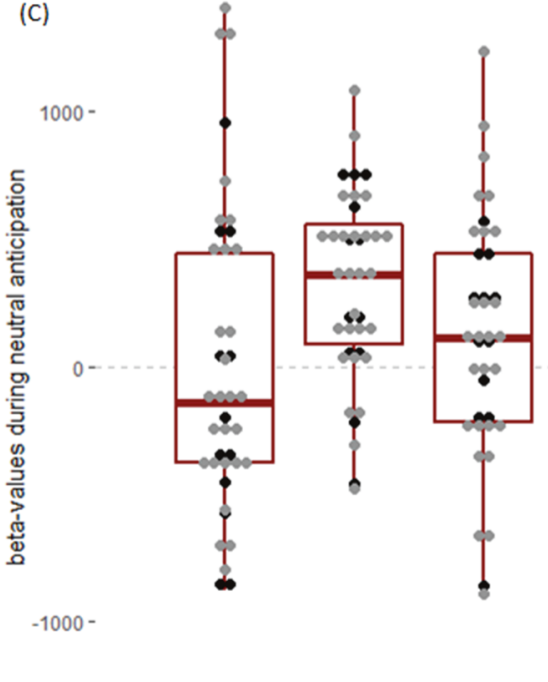

ADHD-only $\quad$ ADHD+SM
Groups
Control

Fig. 1 Whole-brain group differences during reward anticipation. (a) Neural activation in right putamen from the f-test contrast for group differences (across ADHD-only, ADHD + SM and control) during reward anticipation; Boxplots with individual $\beta$-values for this cluster during (b) reward anticipation (i.e., reward cues; $p=0.027$ ) \& (c) neutral anticipation contrast (neutral cues; non-significant in the whole-brain analysis; black dots for participants with $\mathrm{FH}-\&$ gray dots for participants with $\mathrm{FH}+$ ).

are summarized in Tables $\mathrm{S} 1-\mathrm{S} 3$ and visually presented in Figs. S3-S5.

The whole-brain analysis showed group differences during reward anticipation in right putamen $(p=0.027)$. Post-hoc comparisons revealed that $\mathrm{ADHD}+\mathrm{SM}$ activated these regions more than ADHD-only $(p=0.002)$ and controls ( $p<0.001$; Fig. 1). Extracted $\beta$-values for this cluster in neutral anticipation (nonsignificant in the whole-brain analysis) are visually presented in Fig. 1c. No significant clusters were found during neutral anticipation. For the contrast reward minus neutral anticipation, we found group differences in a cluster in left lateral occipital cortex $(p<0.001)$, with decreased activation for ADHD + SM compared to ADHD-only $(p=0.002)$ and controls $(p=0.002$; Fig. S6).

During reward outcome, there were group differences in clusters in right central opecular cortex, pre- and post-central gyri $(p<0.001)$ and in left pre- and post-central gyri $(p<0.001)$. Posthoc comparisons showed decreased activation in ADHD-only ( $p<$ $0.019)$ and ADHD + SM $(p<0.001)$ compared to controls (Fig. S7). We also found group differences in a cluster in right frontal pole and orbitofrontal cortex (OFC; $p=0.004)$, with increased activation for ADHD-only $(p<0.001)$ and ADHD + SM $(p=0.003)$ compared to controls (Fig. 2). During neutral outcome, there were group differences in right frontal pole $(p=0.036)$, with increased activation in ADHD + SM compared to ADHD-only $(p=0.001)$ and controls ( $p=0.013 ;$ Fig. 3 ). No significant clusters were found during reward minus neutral outcome. More detailed results from the fMRI analysis can be found in Tables S1-S5 and Figs. S6-S8.
$R O I$ analysis. The ROI analysis showed no group differences in bilateral NAcc activity during reward minus neutral anticipation $(p=0.23)$ or outcome $(p=0.41$; Fig. S8).

Sensitivity analyses. Group differences of the main analysis remained significant after controlling for additional covariates. Results are summarized in Supplement 2.

Post-hoc exploratory analysis. We found distinct effects of scores in the uncaring ICU scale in ADHD-only versus ADHD + SM (uncaring scores $\times A D H D$ groups; $p=0.001, B=-126.97$ ). This was driven by a positive association in ADHD + SM $(p=0.004, \mathrm{~B}=$ 80.88 ), but not in ADHD-only ( $p=0.174, \mathrm{~B}=-45.66$; Fig. S9).

\section{DISCUSSION}

This study investigated effects of substance misuse on rewardprocessing in ADHD, using whole-brain $\mathrm{fMRI}$ analysis. To our knowledge, the current study is the first to provide evidence for distinct neural activation profiles for ADHD patients with substance misuse, compared to ADHD patients without substance misuse and healthy controls during a reward-processing task. During reward anticipation, ADHD + SM patients showed increased neural responses in right putamen (i.e., dorsal striatum) compared to the other two groups. We also found a positive association between scores on CU traits (uncaring scale) and activation in dorsal striatum for ADHD + SM patients. There were no significant group differences during neutral anticipation. 


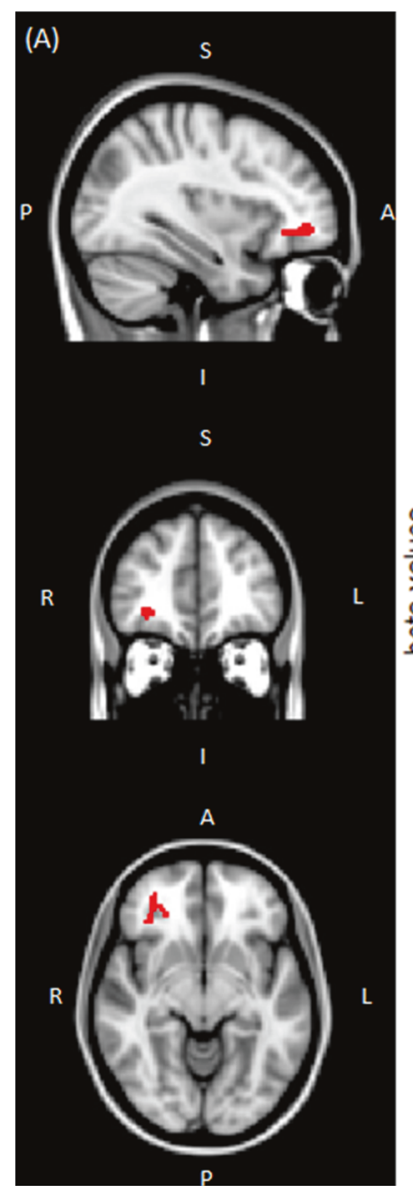

(B)
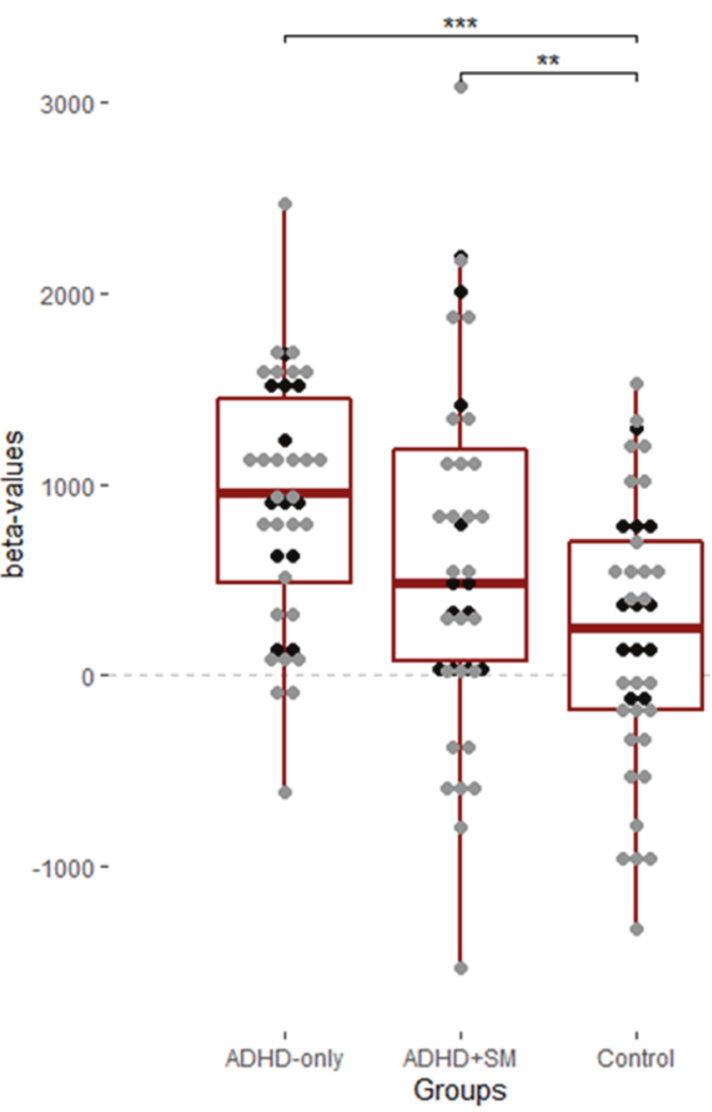

Fig. 2 Whole-brain group differences during reward outcome. (a) Neural activation in right frontal pole and orbitofrontal cortex (OFC) from the $f$-test contrast for group differences (across ADHD-only, ADHD + SM and control) during reward outcome (i.e., reward hits minus reward misses; $p=0.004$ ); (b) Boxplots with individual $\beta$-values for this cluster (black dots for participants with FH- \& gray dots for participants with $\mathrm{FH}+$ ).

During reward minus neutral anticipation, the whole-brain analysis revealed hypoactivation in left lateral occipital cortex in ADHD + SM compared to the other groups, while the ROI analysis showed no group differences in bilateral NAcc. During reward outcome, the ADHD groups showed hypoactivation in pre-, post-central gyri and central opercular cortex and hyperactivation in frontal pole and OFC compared to controls. During neutral outcome, ADHD + SM showed hyperactivation in frontal pole compared to the other groups. During reward minus neutral outcome, both whole-brain and ROI analyses showed no group differences.

Increased neural activation in reward-related areas during reward and neutral conditions, combined with shorter RTs regardless of the condition in $\mathrm{ADHD}+\mathrm{SM}$ implicates distinct condition-independent neural and behavioral correlates in this group. We speculate that reward contingencies affected neural activation and behavior in ADHD + SM patients throughout the task, regardless of the reward condition. This might reflect a general deficit in contingency learning, which subsequently hampers the understanding of the cue-outcome association in a reward task, independent of condition. Moreover, mesolimbic dopamine reward circuit was found to respond to high task demands in the absence of reward, possibly adapting neural resources to changing task requirements [64]. An alternative explanation would thus lie in impaired attentional processing of anticipatory cues in ADHD + SM. Examining neural and behavioral profiles of ADHD patients with substance misuse with tasks that do not include reward contingencies could clarify whether differences observed in this study are task-dependent.
Striatum is one of the core nodes of the mesolimbic dopaminergic pathway and has previously been implicated in reward-processing deficits in both ADHD and (problematic) substance use [15]. Striatal responses to reward anticipation and outcome are thought to represent reward prediction and reward prediction errors (i.e., differences between predicted and received rewards), respectively [65]. Thus, striatal hyperactivation during reward anticipation in ADHD + SM patients might reflect an inflated perceived probability of reward. Moreover, hypoactivation in motor/sensory cortices observed here during reward outcome have been previously linked to reward prediction errors [66]. This might reflect strong reward expectations in ADHD + SM patients, with subsequent small reward prediction error when receiving a reward. Indeed, smaller reward prediction error signal is thought to follow higher reward probabilities $[67,68]$. It would be interesting for future studies to directly measure reward prediction errors and further clarify underlying abnormalities in this population [69].

Despite hypoactivation in motor/sensory cortices, we observed hyperactivation in frontal pole and OFC in both ADHD groups during reward outcome. OFC was found to reflect reward prediction, and not reward prediction errors [70]. OFC is also thought to encode the subjective value of positive outcomes, while frontal pole monitors and evaluates decisions after reward presentation. Moreover, the NAcc ROI analysis showed no group differences and large variability within ADHD + SM group (Fig. S8). Distinct activation patterns across nodes of the reward circuit strongly encourage future studies to use whole-brain analysis approaches without focusing solely on striatum. 


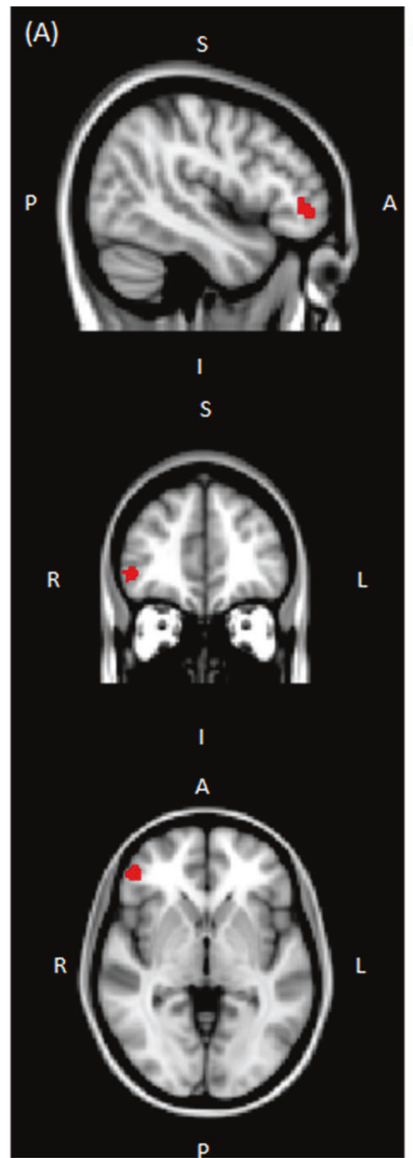

(B)

$6000-$
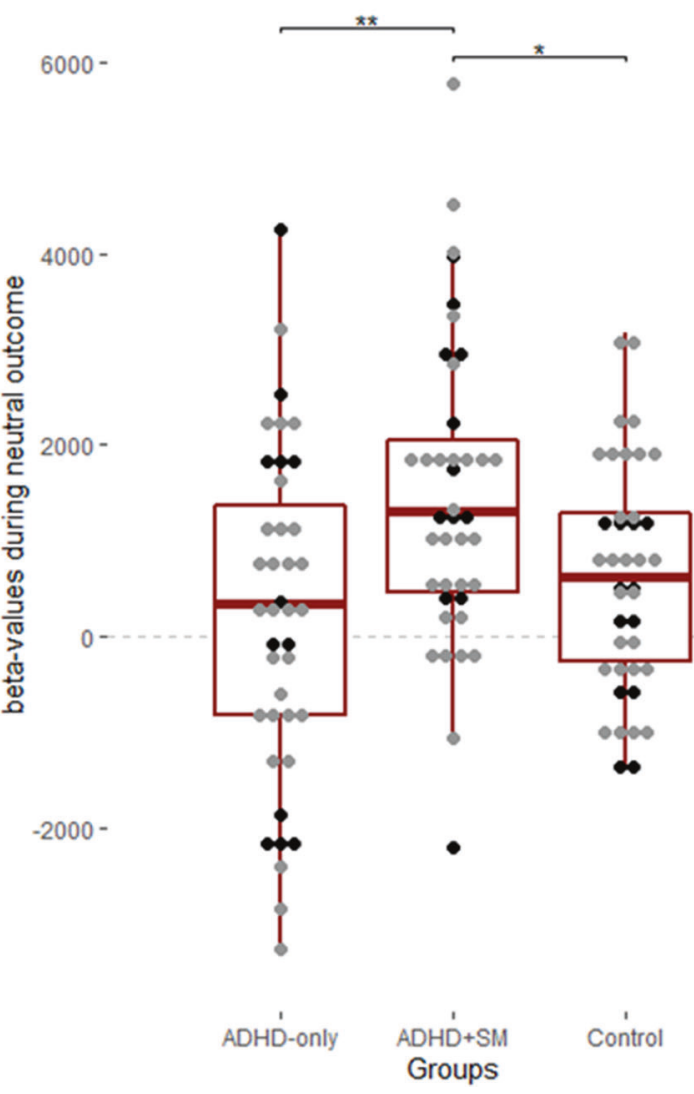

Fig. 3 Whole-brain group differences during neutral outcome. (a) Neural activation in right frontal pole from the $f$-test contrast for group differences (across ADHD-only, ADHD $+\mathrm{SM}$ and control) during neutral outcome (i.e., neutral hits minus neutral misses; $p=0.036$ ); (b) Boxplots with individual $\beta$-values for this cluster (black dots for participants with $\mathrm{FH}-\&$ gray dots for participants with $\mathrm{FH}+$ ).

Increased striatal activation during reward anticipation observed in ADHD + SM patients is not in line with our hypothesis. Although a considerable number of ADHD and SUD studies indicated blunted striatal response to reward anticipation as part of the disorder-specific reward-processing deficits, literature findings show inconsistencies [21, 23, 24, 27]. Our results revealed distinct reward-processing deficits between ADHD patients with and without substance misuse, which might explain part of the inconsistencies observed in the ADHD literature. In fact, our dataset is part of that used by Von Rhein et al. that found nonsignificant VS hyperactivation during reward anticipation for ADHD patients compared to controls, without taking the presence of substance misuse into account [24]. Our results thus suggest that findings of Von Rhein et al. were mainly driven by the presence of ADHD patients with substance misuse. However, data on substance use for parents and participants that were used in the design of the present study were not available for the whole sample of Von Rhein et al. This does not allow us to directly test this assumption in the whole sample of the previous publication.

Striatal hyperactivation resulting from substance misuse is also in contrast with a large body of literature in SUD that indicates VS hypoactivation during reward anticipation [27]. It is important to note that participants with substance misuse in our study did not meet the criteria for SUD, but rather demonstrated substance misuse for the past six months. This might reflect distinct alterations in younger-novice users, compared to chronicallyaddicted older patients that are primarily included in SUD literature. Chronic substance use is believed to gradually lower reward circuit's sensitivity to non-drug incentives [71], but alterations within the reward sensitivity in novice users is less clear. Yet, striatal hypoactivation was previously observed in adolescent smokers [29]. Future studies that will shed light on reward-processing in novice users are needed. Our results are however consistent with prior work by Filbey et al. in chronic cannabis users with comorbid alcohol use [72], and with work by Just et al. that reported putamen hyperactivation as a result of regular stimulant use in a non-ADHD sample (accounting for familial risk) [73].

Elevated striatal activity during reward anticipation in ADHD + SM patients is in line with findings of Yau et al. in children of patients with alcohol use disorder (AUD) with and without problematic drinking behavior. They observed elevated VS activity during reward anticipation for problem drinkers compared to participants with no drinking habits and a positive correlation between VS activity and externalizing behaviors [74]. Moreover, compatible with our results, a recent meta-analysis revealed putamen hyperactivation in adolescents at high SUD risk [75]. Interestingly, these results were no longer significant when studies with comorbid externalizing psychiatric disorders were excluded [75]. Increased VS response to reward anticipation was also found to predict early substance use initiation in children of patients with AUD [30]. This is compatible with early-onset substance use in ADHD $[4,9]$ and the characteristics of our sample.

Notably, converging theories on AUD suggest the existence of two distinct AUD subtypes-Type A/I and Type B/II-that differ in age of onset, severity of substance use, FH load of AUD and amount and severity of psychiatric comorbidities, with a particular focus on the presence of antisocial behavior and personality traits in Type B/II [76-78]. Considering these theories, we speculate that striatal hyperactivation during reward anticipation is not ADHD-specific, 
but is rather present in a subgroup of SUD patients (i.e., Type B/II) that starts substance use at an earlier age and is characterized by more severe SUD, more (severe) psychiatric comorbidities and high levels of antisocial behavior. Indeed, as described by Luijten et al., studies whose samples included SUD patients with one or more psychiatric comorbidities showed less consistent VS hypoactivation [27]. Moreover, antisocial and psychopathic personality traits have previously been associated with VS and OFC hyperactivation during reward anticipation [79-81].

In line with our speculation, ADHD + SM were characterized by higher CU traits (Table 1). Moreover, the exploratory analysis on $\mathrm{CU}$ traits suggested a unique association between $\mathrm{CU}$ traits and reward anticipation in ADHD + SM patients (Fig. S9). Interestingly, $C D$ was previously found to have a mediating role in the association between ADHD to AUD [82]. Future studies should investigate whether $C D$ or $C U$ traits mediate this relationship through altered reward sensitivity at endophenotypic level. Moreover, the increased severity of SUD and the presence of antisocial behavior in Type B/II might lead to difficulties in the recruitment of these participants in studies. VS hypoactivation that has been repeatedly reported in SUD studies might thus reflect a selection bias towards a subgroup with later onset of substance use and different patterns of SUD (i.e., Type A/I).

Some strengths and limitations should be considered in the interpretation of our findings. First, an important strength of our study lies in the design that allowed us to disentangle substance use trait and state effects. The detailed data on parental SUD and the matching with $\mathrm{FH}$ we performed reassured that we measured deficits resulting from substance misuse rather than preexisting abnormalities. It would be interesting for future studies to examine the presence of family history-related deficits in reward-processing in ADHD, preferably in samples with minimal or no substance use. We also ensured that the groups did not differ in other potential confounding variables (i.e., age, sex for ADHD groups only, DBD, ADHD medication) and subsequently performed sensitivity analysis with these variables as additional covariates. However, after matching the groups with $\mathrm{FH}$, the largest part of each group consisted of participants at high risk for SUD (i.e., 28 with $\mathrm{FH}+, 12$ with $\mathrm{FH}$-). Importantly, studies with individuals with $\mathrm{FH}+$ showed VS hypoactivation compared to those with $\mathrm{FH}$ - or no differences between groups during reward anticipation $[74,83]$. To make sure that such trait effects do not interfere with group differences in striatal activation, we included $\mathrm{FH}$ as covariate in all our analyses. Results were also plotted distinguishing participants based on FH (Fig. 1). Another limitation of our sample was sex imbalance. This resulted from ADHD + SM that consisted of 32 males and 8 females. To account for potential sex effects, ADHD-only and ADHD + SM were perfectly matched with sex, but the data did not allow us to also match controls with this variable. Yet, sex imbalance is in accordance with male-tofemale ratios found in the ADHD literature [84]. In addition, the present study did not include an SM-only group (i.e., without ADHD), which would allow a direct comparison between SM-only and ADHD + SM. Future studies should address this issue, by recruiting SUD/SM patients with and without ADHD. Another limitation is that the available instrument for substance use included information about the frequency, but not severity, of alcohol, drug, and nicotine use. This allowed us to examine substance misuse, but not SUD in participants. Future studies should include more detailed instruments on substance use to be able to investigate the relationship between substance use and reward-processing in ADHD in more detail. Moreover, the current study included a modified version of the traditional MID task that might challenge comparison of our findings with those of other studies. In detail, the task included a low reward magnitude and low hit probability, compared to other studies. Literature shows more robust striatal responses for higher than lower reward magnitude [85]. Yet dopaminergic midbrain neurons are thought to code for the relative reward value, depending on the context, in which the reward is presented [86]. Low hit probability might have affected the perceived difficulty of the task, resulting in frustration and surprise, instead of anticipation and receipt of reward. However, similar to reward magnitude, hit probability is coded relatively, as a function of the context. Thus, as the task did not combine multiple reward levels or blocks with varying hit probabilities, we believe that differences in the task parameters have not significantly affected our results [24]. Task-related limitations can also be found in Von Rhein et al. [24]. Future studies should examine whether VS hyperactivation during reward anticipation in ADHD + SM patients is confirmed in MID tasks with higher reward magnitude and hit probability. Given the relative representation of reward value as a function of context, future studies would also benefit from inclusion of mixed reward and loss trials.

Taken together, we provide evidence for distinct rewardprocessing deficits in ADHD patients with substance misuse compared to those without. This suggests that substance misuse effects and variability of substance misuse prevalence across ADHD studies might have contributed to inconsistent findings in the literature. Future studies in ADHD should take presence of substance use into account either during participant recruitment or during statistical analysis. The observed reward-related neural activity profile of ADHD + SM patients is in contrast with a large body of literature in SUD and recreational substance use. We argue that these inconsistencies with the SUD literature might be explained by the existence of distinct SUD subtypes with differences in age of onset, presence of psychiatric comorbidities and antisocial traits. Future studies should compare rewardprocessing between SUD patients with and without ADHD in relation to age of onset of substance misuse and psychopathic traits.

\section{FUNDING AND DISCLOSURE}

This work was supported by VENI Grant 916.15.101 (to AFAS) from the Netherlands Organisation for Scientific Research (NWO) and grants from Radboud University Nijmegen Medical Center. The NeurolMAGE project was supported by NIH Grant R01MH62873 (to Stephen V. Faraone), NWO Large Investment Grant 1750102007010 and ZonMw Grant 60-60600-97-193 (to JKB), grants from Radboud University Nijmegen Medical Center, University Medical Center Groningen and Accare, and VU University Amsterdam and an unrestricted grant from Shire Pharmaceuticals (to Stephen V. Faraone). The work is further supported by the Horizon2020 programme of the European Union (grant number 667302 for the Comorbid Conditions of Attentiondeficit/ Hyperactivity Disorder (CoCA) consortium). JKB has been a consultant to/member of advisory board of and/or speaker for Janssen Cilag BV, Eli Lilly, Roche, Medice, Takeda/Shire, Novartis and Servier. He is not an employee or a stock shareholder of any of these companies. He has no other financial or material support, including expert testimony, patents, and royalties. The other authors declare no biomedical financial interests or potential conflicts of interest.

\section{AUTHOR CONTRIBUTIONS}

MP has contributed to the design of project, the data analysis and interpretation of the results and the writing of the manuscript. She has also agreed to be accountable for all aspects of the work in ensuring that questions related to the accuracy or integrity of any part of the work are appropriately investigated and resolved. DVR has contributed to the design of the work, the data acquisition and analysis, the interpretation of the results, the writing and revision of the manuscript. $A B$ has contributed to the design of the project, the interpretation of the results and the revision of the manuscript for important intellectual content. RC has contributed to the data analysis and the revision of the manuscript for important intellectual content. ML has contributed to the revision of the manuscript 
for important intellectual content. AHS has contributed to the design of the work, the interpretation of the results and the revision of the manuscript for important intellectual content. JKB has contributed to the design of the project, the interpretation of the results and the revision of the manuscript for important intellectual content. AFAS has contributed to the conception and design of the project, the data analysis, the interpretation of the results, the writing and revision of the manuscript for important intellectual content. All authors have approved the publication of the manuscript.

\section{ADDITIONAL INFORMATION}

Supplementary Information accompanies this paper at (https://doi.org/10.1038/ s41386-020-00896-1).

Publisher's note Springer Nature remains neutral with regard to jurisdictional claims in published maps and institutional affiliations.

\section{REFERENCES}

1. Faraone SV, Biederman J, Mick E. The age-dependent decline of attention deficit hyperactivity disorder: A meta-analysis of follow-up studies. Psychol Med. 2006;36:159-65.

2. Polanczyk G, Rohde LA. Epidemiology of attention-deficit/hyperactivity disorder across the lifespan. Curr Opin Psychiatry. 2007;20:386-92.

3. Molina BSG, Pelham WE. Attention-deficit/hyperactivity disorder and risk of substance use disorder: developmental considerations, potential pathways, and opportunities for research. Annu Rev Clin Psychol. 2014;10:607-39.

4. Lee SS, Humphreys KL, Flory K, Liu R, Glass K. Prospective association of childhood attention-deficit/hyperactivity disorder (ADHD) and substance use and abuse/dependence: a meta-analytic review. Clin Psychol Rev. 2011;31:328-41.

5. Ilbegi S, Groenman AP, Schellekens A, Hartman CA, Hoekstra PJ, Franke B, et al. Substance use and nicotine dependence in persistent, remittent, and late-onset ADHD: a 10-year longitudinal study from childhood to young adulthood. J Neurodev Disord. 2018;10:1-8.

6. Biederman J, Wilens T, Mick E, Milberger S, Spencer TJ, Faraone SV. Psychoactive substance use disorders in adults with attention deficit hyperactivity disorder (ADHD): effects of ADHD and psychiatric comorbidity. Am J Psychiatry. 1995;152:1652-8.

7. Sizoo $B$, van den Brink W, Koeter $M$, van Eenige MG, van Wijngaarden-Cremers $P$, van der Gaag RJ. Treatment seeking adults with autism or ADHD and co-morbid Substance Use Disorder: Prevalence, risk factors and functional disability. Drug Alcohol Depend. 2010;107:44-50

8. Wittchen HU, Jacobi F, Rehm J, Gustavsson A, Svensson M, Jönsson B, et al. The size and burden of mental disorders and other disorders of the brain in Europe 2010. Eur. Neuropsychopharmacol. 2011;21:655-79.

9. Wilens TE. Impact of ADHD and its treatment on substance abuse in adults. J Clin Psychiatry. 2004;65:38-45.

10. Castells X, Ramos-Quiroga JA, Rigau D, Bosch R, Nogueira M, Vidal X, et al. Efficacy of methylphenidate for adults with attention-deficit hyperactivity disorder: a meta-regression analysis. CNS Drugs. 2011;25:157-69.

11. American Psychiatric Association. Diagnostic and statistical manual of mental disorders (DSM-5 ${ }^{\oplus}$ ). American Psychiatric Publication; 2013.

12. Faraone SV, Asherson P, Banaschewski T, Biederman J, Buitelaar JK, RamosQuiroga JA, et al. Attention-deficit/hyperactivity disorder. Nat Rev Dis Prim. 2015;1:15020.

13. Coffey SF, Gudleski GD, Saladin ME, Brady KT. Impulsivity and rapid discounting of delayed hypothetical rewards in cocaine-dependent individuals. Exp Clin Psychopharmacol. 2003;11:18-25.

14. DL Von, Bassani DG, Fuchs SC, Szobot CM, Pechansky F. Impulsivity, age of first alcohol use and substance use disorders among male adolescents: a population based case-control study. Addiction. 2008;1198-205. https://doi.org/10.1111/ j.1360-0443.2008.02223.x

15. Adisetiyo V, Gray KM. Neuroimaging the neural correlates of increased risk for substance use disorders in attention-deficit/hyperactivity disorder-A systematic review. Am. J. Addict. 2017;26:99-111.

16. Zuckerman M Sensation seeking (psychology revivals): beyond the optimal level of arousal. Psychology Press; 2014.

17. Haber SN, Knutson B. The reward circuit: linking primate anatomy and human imaging. Neuropsychopharmacology. 2010;35:4-26.

18. Galvan A. Adolescent development of the reward system. Front Hum Neurosci. 2010;4:6.

19. Greven CU, Bralten J, Mennes M, O'Dwyer L, van Hulzen KJ, Rommelse N, et al. Developmentally stable whole-brain volume reductions and developmentally sensitive caudate and putamen volume alterations in those with attention- deficit/hyperactivity disorder and their unaffected siblings. JAMA Psychiatry. 2015;1-10. https://doi.org/10.1001/jamapsychiatry.2014.3162.

20. Shaw P, De Rossi P, Watson B, Wharton A, Greenstein D, Raznahan A, et al. Mapping the development of the basal ganglia in children with attention-deficit/ hyperactivity disorder. J Am Acad Child Adolesc Psychiatry. 2014;53:780-9.

21. Plichta $M M$, Scheres $A$. Ventral-striatal responsiveness during reward anticipation in ADHD and its relation to trait impulsivity in the healthy population: a metaanalytic review of the fMRI literature. Neurosci Biobehav Rev. 2014;38:125-34.

22. van Hulst BM, de Zeeuw P, Bos DJ, Rijks Y, Neggers SF, Durston S, et al. Children with $A D H D$ symptoms show decreased activity in ventral striatum during the anticipation of reward, irrespective of ADHD diagnosis. J Child Psychol Psychiatry Allied Discip. 2017;58:206-14.

23. Hägele $C$, Schlagenhauf $F$, Rapp $M$, Sterzer $P$, Beck A, Bermpohl F, et al. Dimensional psychiatry: Reward dysfunction and depressive mood across psychiatric disorders. Psychopharmacology (Berl). 2015;232:331-41.

24. von Rhein D, Cools R, Zwiers MP, van der Schaaf M, Franke B, Luman $M$, et al. Increased neural responses to reward in adolescents and young adults with attention-deficit/hyperactivity disorder and their unaffected siblings. J Am Acad Child Adolesc Psychiatry. 2015;54:394-402.

25. Paloyelis Y, Mehta MA, Faraone SV, Asherson P, Kuntsi J. Striatal sensitivity during reward processing in attention-deficit/hyperactivity disorder. J Am Acad Child Adolesc Psychiatry. 2012;51:722-32.

26. Scheres A, Milham MP, Knutson B, Castellanos FX. Ventral striatal hyporesponsiveness during reward anticipation in attention-deficit/hyperactivity disorder. Biol Psychiatry. 2007;61:720-4.

27. Luijten M, Schellekens AF, Kühn S, Machielse MWJ, Sescousse G. Disruption of reward processing in addiction. JAMA Psychiatry. 2017;74:387.

28. Schouw MLJ, De Ruiter MB, Kaag AM, van den Brink W, Lindauer RJL, Reneman L, et al. Dopaminergic dysfunction in abstinent dexamphetamine users: Results from a pharmacological fMRI study using a reward anticipation task and a methylphenidate challenge. Drug Alcohol Depend. 2013;130:52-60.

29. Peters J, Bromberg U, Schneider S, Brassen S, Menz M, Banaschewski T, et al. Lower ventral striatal activation during reward anticipation in adolescent smokers. Am J Psychiatry. 2011;168:540-9.

30. Cope LM, Martz ME, Hardee JE, Zucker RA, Heitzeg MM. Reward activation in childhood predicts adolescent substance use initiation in a high-risk sample. Drug Alcohol Depend. 2019;194:318-25.

31. Dallery J, Locey ML. Effects of acute and chronic nicotine on impulsive choice in rats. Behav pharmacol. 2000; 15-23.

32. Eppolito AK, France CP, Gerak LR. Effects of acute and chronic morphine on delay discounting in pigeons. J Exp Anal Behav. 2013;99:277-89.

33. Groman SM, Lee B, Seu E, James AS, Feiler K, Mandelkern MA, et al. Dysregulation of D2-mediated dopamine transmission in monkeys after chronic escalating methamphetamine exposure. J Neurosci. 2012;32:5843-52.

34. Paine TA, Dringenberg HC, Olmstead MC. Effects of chronic cocaine on impulsivity: relation to cortical serotonin mechanisms. Behav Brain Res. 2003;147: 135-47.

35. Liu S, Heitz RP, Bradberry CW. A touch screen based stop signal response task in rhesus monkeys for studying impulsivity associated with chronic cocaine selfadministration. J Neurosci Methods. 2009;177:67-72.

36. Kolokotroni $\mathrm{KZ}$, Rodgers RJ, Harrison AA. Effects of chronic nicotine, nicotine withdrawal and subsequent nicotine challenges on behavioural inhibition in rats. Psychopharmacology (Berl). 2012;219:453-68.

37. Setlow B, Mendez IA, Mitchell MR, Simon NW. Effects of chronic administration of drugs of abuse on impulsive choice (delay discounting) in animal models. Behav Pharmacol. 2009;20:380-9.

38. Belin D, Mar AC, Dalley JW, Robbins TW, Everitt BJ. High impulsivity predicts the switch to compulsive cocaine-taking. Science. 2008;320:1352-5.

39. Ortal S, van de Glind G, Johan F, Itai B, Nir Y, lliyan I, et al. The role of different aspects of impulsivity as independent risk factors for substance use disorders in patients with ADHD: a review. Curr. Drug Abuse Rev. 2015;8:119-33.

40. Edwards AC, Kendler KS. Twin study of the relationship between adolescent attention-deficit/hyperactivity disorder and adult alcohol dependence. J Stud Alcohol Drugs. 2012;73:185-94.

41. Blair RJR. The neurobiology of psychopathic traits in youths. Nat. Rev. Neurosci. 2013;14:786-99.

42. Frick PJ, Moffitt TE. A proposal to the DSM-V childhood disorders and the ADHD and disruptive behavior disorders work groups to include a specifier to the diagnosis of conduct disorder based on the presence of callous-unemotional traits. Washington, DC. Am Psychiatr Assoc. 2010;1-36.

43. von Rhein D, Mennes M, van Ewijk $H$, Groenman AP, Zwiers MP, Oosterlaan J, et al. The NeuroIMAGE study: a prospective phenotypic, cognitive, genetic and MRI study in children with attention-deficit/hyperactivity disorder. Design and descriptives. Eur Child Adolesc Psychiatry. 2014. https://doi.org/10.1007/s00787014-0573-4. 
44. Kaufman J, Birmaher B, Brent D, Rao UMA, Flynn C, Moreci P, et al. Schedule for affective disorders and schizophrenia for school-age children-present and lifetime version (K-SADS-PL): initial reliability and validity data. J Am Acad Child Adolesc Psychiatry. 1997;36:980-8.

45. Conners CK, Sitarenios G, Parker JDA, Epstein JN. Revision and restandardization of the conners teacher rating scale (CTRS-R): factor structure. Reliability Criterion Val. 1998;26:279-91.

46. Conners CK, Erhardt D, Epstein JN, Parker JDA, Sitarenios G, Sparrow E. Selfratings of $A D H D$ symptoms in adults I: Factor structure and normative data. J Atten Disord. 1999;3:141-51.

47. Loeber R, Wung P, Keenan K, Giroux B, Stouthamer-Loeber M, Van Kammen WB, et al. Developmental pathways in disruptive child behavior. Dev Psychopathol. 1993;5:103-33.

48. Elliott DS, Huizinga D, Ageton SS Explaining delinquency and drugs use. Beverly Hills, CA: Sage.; 1985.

49. Schippers GM, Broekman TG, Buchholz A, Koeter MWJ. Van Den Brink W. Measurements in the Addictions for Triage and Evaluation (MATE): An instrument based on the World Health Organization family of international classifications. Addiction. 2010;105:862-71.

50. Saunders JB, Aasland OG, Babor TF, De La Fuente JR, Grant M. Development of the alcohol use disorders identification test (AUDIT): WHO collaborative project on early detection of persons with harmful alcohol consumption-Il. Addiction. 1993;88:791-804

51. Gavin DR, Ross HE, Skinner HA. Diagnostic validity of the DAST in the assessment of DSM-III drug disorders. Br J Addict. 1989;84:301-7.

52. Heatherton TF, Kozlowski LT, Frecker RC, Fagerstrom K-O. The Fagerström test for nicotine dependence: a revision of the fagerstrom tolerance questionnaire. $\mathrm{Br}$. J. Addict. 2018;86:1119-27.

53. Sobell LC, Brown J, Leo GI, Sobell MB. The reliability of the alcohol timeline followback when administered by telephone and by computer. Drug Alcohol Depend. 1996;42:49-54.

54. RStudio Team. RStudio: Integrated Development for R. 2019. Boston, MA: RStudio Inc. http://www.rstudio.com/.

55. Ho DE, Imai K, King G, Stuart EA. Matchlt: nonparametric preprocessing for parametric causal inference. J Stat Softw. 2011;42. https://doi.org/10.18637/jss. v042.i08.

56. Kimonis ER, Frick PJ, Skeem JL, Marsee MA, Cruise K, Munoz LC, et al. Assessing callous-unemotional traits in adolescent offenders: validation of the Inventory of Callous-Unemotional Traits. Int J Law Psychiatry. 2008;31:241-52.

57. Byrd Amy L, Kahn Rachel E, Pardini DA. A validation of the inventory of callousunemotional traits in a community sample of young adult males. J Psychopathol Behav Assess. 2014;35:1-26.

58. Gow RV, Vallee-Tourangeau F, Crawford MA, Taylor E, Ghebremeskel K, Bueno AA et al. Omega-3 fatty acids are inversely related to callous and unemotional traits in adolescent boys with attention de fi cit hyperactivity disorder. Prostaglandins Leukot Essent Fat Acids. 2013;88:411-8.

59. Hoogman $M$, Aarts $E$, Zwiers $M$, Slaats-Willemse $D$, Naber $M$, Onnink $M$, et al. Nitric oxide synthase genotype modulation of impulsivity and ventral striatal activity in adult ADHD patients and healthy comparison subjects. Am J Psychiatry. 2011;168:1099-106.

60. Knutson B, Fong GW, Adams CM, Varner JL, Hommer D. Dissociation of reward anticipation and outcome with event-related fMRI. Neuroreport. 2001;12:3683-7.

61. Smith SM, Jenkinson M, Woolrich MW, Beckmann CF, Behrens TE, Johansen-Berg $\mathrm{H}$, et al. Advances in functional and structural MR image analysis and implementation as FSL. Neuroimage. 2004;23:S208-19.

62. Jenkinson M, Beckmann CF, Behrens TEJ, Woolrich MW, Smith SM. FSL. Neuroimage. 2012;62:782-90.

63. Smith SS, Newman JP. Alcohol and drug abuse-dependence disorders in psychopathic and nonpsychopathic criminal offenders. J. Abnorm. Psychol. 1990;99: 430-9.
64. Boehler CN, Hopf JM, Krebs RM, Stoppel CM, Schoenfeld MA, Heinze HJ, et al. Task-load-dependent activation of dopaminergic midbrain areas in the absence of reward. 2011;31:4955-61.

65. Schultz W. Updating dopamine reward signals. Curr Opin Neurobiol. 2013;23: 229-38.

66. Cservenka A, Courtney KE, Ghahremani DG, Hutchison KE, Ray LA. Development, initial testing and challenges of an ecologically valid reward prediction error FMRI task for alcoholism. Alcohol Alcohol. 2017;52:617-24.

67. Fiorillo CD, Tobler PN, Schultz W. Discrete coding of reward probability and uncertainty by dopamine neurons. Science. 2003;299:1898-902.

68. Linnet J. Neurobiological underpinnings of reward anticipation and outcome evaluation in gambling disorder. Front Behav Neurosci. 2014;8:1898-902.

69. Cao Z, Bennett M, Orr C, Icke I, Banaschewski T, Barker GJ, et al. Mapping adolescent reward anticipation, receipt, and prediction error during the monetary incentive delay task. Hum Brain Mapp. 2019;40:262-83.

70. Stalnaker TA, Liu T, Takahashi YK, Schoenbaum G. Orbitofrontal neurons signal reward predictions, not reward prediction errors. Neurobiol Learn Mem. 2018;153:137-43.

71. Goldstein RZ, Volkow ND. Dysfunction of the prefrontal cortex in addiction: neuroimaging findings and clinical implications. 2011;12:652-69.

72. Filbey FM, Dunlop J, Myers US. Neural effects of positive and negative incentives during marijuana withdrawal. PloS one. 2013;8.

73. Just AL, Meng C, Smith DG, Bullmore ET, Robbins TW, Ersche KD, et al. Effects of familial risk and stimulant drug use on the anticipation of monetary reward: an fMRI study. Transl Psychiatry. 2019;9.

74. Yau WYW, Zubieta JK, Weiland BJ, Samudra PG, Zucker RA, Heitzeg MM, et al. Nucleus accumbens response to incentive stimuli anticipation in children of alcoholics: relationships with precursive behavioral risk and lifetime alcohol use. J Neurosci. 2012;32:2544-51.

75. Tervo-clemmens B, Quach A, Calabro FJ, Foran W, Luna B. Neurolmage metaanalysis and review of functional neuroimaging differences underlying adolescent vulnerability to substance use. Neuroimage. 2020;209:116476.

76. Cloninger CR, Sigvardsson S, Bohman M. Childhood personality predicts alcohol abuse in young adults. Alcohol Clin Exp Res. 1988;12:494-505.

77. Hansen WB, Flay BR, Weber MD, Johnson CA, Graham JW. Evidence for two paths of alcohol use onset in adolescents. Addict Behav. 1989;14:399-408.

78. Fitzgerald HE, Ellis DA, Sanford K, Zucker RA, Bingham CR. Other evidence for at least two alcoholisms II: Life course variation in antisociality and heterogeneity of alcoholic outcome. Dev Psychopathol. 2009;8:831.

79. Murray L, Waller R, Hyde LW. A systematic review examining the link between psychopathic personality traits, antisocial behavior, and neural reactivity during reward and loss processing. Personal Disord Theory, Res Treat. 2018;9:497-509.

80. Buckholtz JW, Treadway MT, Cowan RL, Woodward ND, Benning SD, Li R, et al. Mesolimbic dopamine reward system hypersensitivity in individuals with psychopathic traits. Nat Neurosci. 2010;13:419-21.

81. Bjork JM, Chen G, Hommer DW. Psychopathic tendencies and mesolimbic recruitment by cues for instrumental and passively obtained rewards. Biol Psychol. 2012;89:408-15.

82. Tuithof M, ten Have M, van den Brink W, Vollebergh W, de Graaf R. The role of conduct disorder in the association between ADHD and alcohol use (disorder). Results from the Netherlands Mental Health Survey and Incidence Study-2. Drug Alcohol Depend. 2012;123:115-21.

83. Bjork JM, Knutson B, Hommer DW. Incentive-elicited striatal activation in adolescent children of alcoholics. Addiction. 2008;103:1308-19.

84. Slobodin O, Davidovitch M. Gender differences in objective and subjective measures of ADHD among clinic-referred children. Front Hum Neurosci. 2019;13: $1-14$

85. Wu CC, Samanez-Larkin GR, Katovich K, Knutson B. Affective traits link to reliable neural markers of incentive anticipation. Neuroimage. 2014;84:279-89.

86. Tobler PN, Fiorillo CD, Schultz W. Adaptive coding of reward value by dopamine neurons. Science. 2005;307:1642-5. 